\title{
Adherence to the National Institute of Clinical Excellence guidance on parenteral nutrition screening is not adequate to improve outcomes
}

\author{
S. P. Omar ${ }^{1}$, R. Vincent ${ }^{1}$, M. O. Elnenaei ${ }^{1}$, C. H. Goo ${ }^{2}$, R. Salota ${ }^{1}$, P. F. Wu ${ }^{3}$, \\ H. L. Delaney ${ }^{1}$ and C. W. Le Roux ${ }^{1}$ \\ ${ }^{1}$ Department of Chemical Pathology, King's College Hospital NHS Foundation Trust, Denmark Hill, London SE5 9RS, UK, \\ ${ }^{2}$ Department of Nutrition \& Dietetics, International Medical University, Kuala Lumpur 57000, Malaysia and ${ }^{3}$ Department \\ of Pharmacy, King's College Hospital NHS Foundation Trust, Denmark Hill, London SE5 9RS, UK
}

\begin{abstract}
Absence of randomised controlled trials to determine clinical practice in parenteral nutrition (PN) meant that a large proportion of the National nutrition guidance recommendations in the UK were based on consensus and expert opinion ${ }^{(1)}$. Aim of the study was to assess outcome measures of PN administration when the national nutrition guidance was adhered to.

The prospective study evaluated patients referred to the multi-disciplinary PN team between 2008 and 2010. Information was obtained from medical notes, electronic patient records, dietetic records, nursing care plans and PN prescription charts. Patients who were treated with enteral nutrition were outside the remit of this study. Patients were divided into two groups: guidance compliant and guidance noncompliant. Outcome measures were duration of PN treatment, total number of PN bags used per patient, length of hospital stay and mortality.

The study included 262 adult patients, aged 54 (42-67) [median (IQR)] years of which 155 were male. Guidance compliant group consisted of 143 and non-compliant group 119 patients. In the compliant group $100 \%$ were screened on initial admission compared to $40 \%$ in the non-compliant $(P<0.001)$. Based on screening criteria $58 \%$ and $78 \%$ were identified as either malnourished or at risk of malnutrition in the compliant and non-compliant groups respectively $(P<0.001)$. In compliant group, of those malnourished or at risk of malnutrition, $100 \%$ were referred for dietetic assessment, but in the non-compliant group this was done in only $14 \%(<0.001)$. However, those in the compliant group received nutritional support earlier than in the non-compliant group; 0 (0-4) days vs 2 (0-5) days $(P=0.005)$. There was no difference in duration of PN treatment, total number of PN bags used per patient and length of hospital stay between the groups (all $P>0.05$ ). All cause mortality within 30-days of initiating PN, in compliant and non-compliant was $33 \%$ and $29 \%$ respectively $(P=0.29)$.

There was no difference in outcome measures of PN administration and mortality between the groups. More studies are needed to generate evidence on which to base nutrition guidance on. Mortality in patients receiving PN is multi-factorial, but better understanding of these causes may help determine what changes to PN practice are required.
\end{abstract}

1. NICE Clinical Guideline 32. Nutrition Support in Adults: Oral Nutrition Support, Enteral Tube Feeding and Parenteral Nutrition. London 2006. 